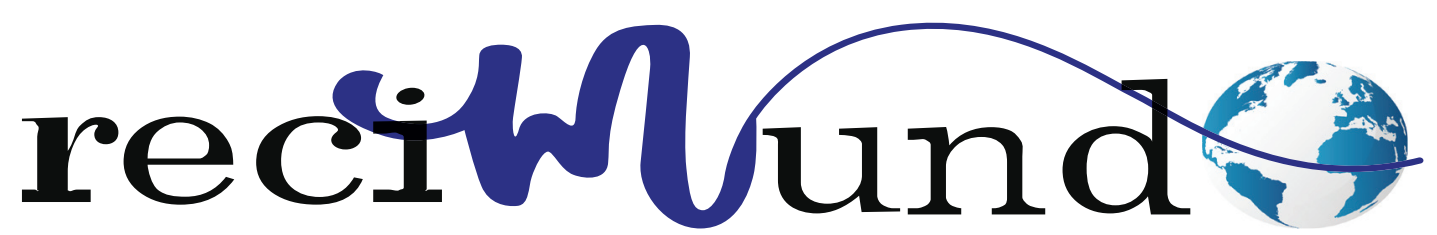

Revista Científica Mundo de la Investigación y el Conocimiento

DOI: 10.26820/recimundo/5.(2).abril.2021.4-15

URL: hhttps://recimundo.com/index.php/es/article/view/1028

EDITORIAL: Saberes del Conocimiento

REVISTA: RECIMUNDO

ISSN: 2588-073X

TIPO DE INVESTIGACIÓN: Artículo de revisión

CÓDIGO UNESCO: 32 Ciencias Médicas

PAGINAS: 4-15

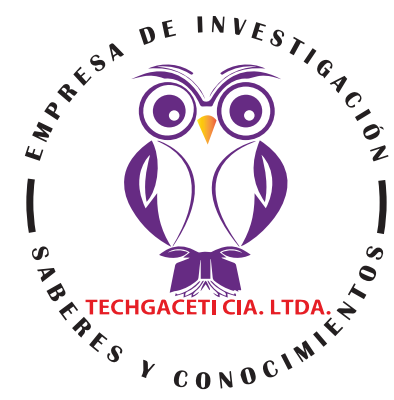

\title{
Coartación aortica, diagnóstico y tratamiento
}

Aortic coarctation, diagnosis and treatment

Coarctação da Aorta, Diagnóstico e Tratamento

Kory Anahi Reinoso Trujillo; Rosana Fabregas Almanza²; Fredy Alexis Mendoza Niño3; Daniela Mindiola Joly ${ }^{4}$

\section{RECIBIDO: 15/01/2021 ACEPTADO: 20/03/2021 PUBLICADO: 01/04/2021}

1. Médico Residente de Cirugía General del Hospital de Especialidades FFAA Nº1; Quito, Ecuador; kory318anahi@gmail. com; (iD https://orcid.org/0000-0002-7191-899X

2. Médico General; Investigador Independiente; Guayaquil, Ecuador; rochyfa@hotmail.com; iD https://orcid.org/0000-00019756-9102

3. Médico adjunto al Departamento de Radiología Hospital del Sarare; fredd0492@hotmail.com; iD https://orcid.org/00000001-8384-4003

4. Médico General; Investigador Independiente; damamijo324@gmail.com; (D) https://orcid.org/0000-0003-1685-6789

\section{CORRESPONDENCIA}

Kory Anahi Reinoso Trujillo

kory318anahi@gmail.com

Quito, Ecuador 


\section{RESUMEN}

La aorta es la arteria más grande y de mayor importancia del cuerpo humano, es la encargada de llevar la sangre rica en oxigeno desde el corazón a todas las demás zonas del organismo. Cuando ocurre un estrechamiento de esta arteria, se produce lo que llaman coartación aortica, ésto hace que el corazón tenga que bombear con más fuerza para poder impulsar la sangre a través de la aorta. Es un tipo de afección congénito que se detecta a edad adulta y se presenta en el conducto arterial. Por medio de una revisión de literatura, se discutirá la epidemiología y etiología del adulto con coartación de la aorta (CoA), describiendo la embriología, anatomía, fisiopatología y presentación clínica para reconocer y diagnosticar adecuadamente a un paciente adulto con CoA. Esta investigación también revisará las pruebas de diagnóstico, el manejo, las intervenciones terapéuticas, incluidos los procedimientos quirúrgicos y percutáneos, y las complicaciones a largo plazo que pueden surgir en un adulto con CoA reparada.

Palabras clave: Cardiopatía congénita, coartación de la aorta, intervención percutánea, dilatación con balón, colocación de stents, cirugía, manejo, complicaciones.

\section{ABSTRACT}

The aorta is the largest and most important artery in the human body, it is responsible for carrying oxygen-rich blood from the heart to all other areas of the body. When a narrowing of this artery occurs, what is called aortic coarctation occurs, this causes the heart to pump with more force to be able to push the blood through the aorta. It is a type of congenital condition that is detected in adulthood and occurs in the ductus arteriosus. Through a literature review, the epidemiology and etiology of the adult with coarctation of the aorta $(\mathrm{COA})$ will be discussed, describing the embryology, anatomy, pathophysiology, and clinical presentation to properly recognize and diagnose an adult patient with CoA. This research will also review diagnostic testing, management, therapeutic interventions, including surgical and percutaneous procedures, and long-term complications that can arise in an adult with repaired CoA.

Keywords: Congenital heart disease, aortic coarctation, percutaneous intervention, balloon dilation, stenting, surgery, management, complications.

\section{RESUMO}

A aorta é a maior e mais importante artéria do corpo humano, ela é responsável por transportar sangue rico em oxigênio do coração para todas as outras áreas do corpo. Quando ocorre um estreitamento desta artéria, o que é chamado de coarctação da aorta, isto faz com que o coração bombeie com mais força para ser capaz de empurrar o sangue através da aorta. É um tipo de condição congênita que é detectada na idade adulta e ocorre no ducto arteriosus. Através de uma revisão da literatura, a epidemiologia e etiologia do adulto com coarctação da aorta (CoA) será discutida, descrevendo a embriologia, anatomia, fisiopatologia e apresentação clínica para reconhecer e diagnosticar adequadamente um paciente adulto com CoA. Esta pesquisa também revisará testes diagnósticos, manejo, intervenções terapêuticas, incluindo procedimentos cirúrgicos e percutâneos, e complicações a longo prazo que podem surgir em um adulto com a CoA reparada.

Palavras-chave: Doença cardíaca congênita, coarctação da aorta, intervenção percutânea, dilatação do balão, stenting, cirurgia, manejo, complicações. 


\section{Introducción}

La coartación de la aorta es un defecto cardíaco congénito. Suele manifestarse como una constricción discreta del istmo aórtico. Sin embargo, es más probable que represente un espectro de estrechamiento aórtico desde esta entidad discreta hasta la hipoplasia tubular, con muchas variaciones observadas entre estos dos extremos. "Los morfólogos sostienen que la hipoplasia tubular, aunque puede coexistir con una coartación discreta, debe considerarse como una entidad separada" (Kenny \& Hijazi, 2011). En raras ocasiones puede haber un espacio entre la aorta torácica ascendente y descendente, conocido como arco aórtico interrumpido.

Las intervenciones pueden ser necesarias en la infancia, sin embargo, los procedimientos pueden ser necesarios más adelante en la vida para la coartación nativa o los pacientes con coartación recurrente.

La coartación de la aorta es la sexta lesión congénita más común y representa del $4 \%$ al $6 \%$ de los nacidos vivos con cardiopatía congénita. Aunque la mayoría de los pacientes tienen un estrechamiento discreto de la aorta torácica en la inserción del conducto arterioso, el espectro anatómico puede variar desde esta entidad discreta hasta la hipoplasia tubular, con muchas variaciones entre estos extremos (Baumgartner, 2015).

A pesar de estas variaciones anatómicas, el efecto del estrechamiento tiene las características comunes de "aumento de la poscarga en el ventrículo izquierdo, exposición de la parte superior del cuerpo a la hipertensión, alteración del flujo en la aorta torácica y disminución de la perfusión en la parte inferior del cuerpo" (Brown, 2013). Dependiendo del equilibrio entre el grado de perturbación del flujo y los mecanismos compensatorios disponibles para superarlo, la presentación clínica puede variar desde el recién nacido críticamente enfermo con insuficiencia cardíaca hasta el niño o adulto asintomático con hipertensión.

La coartación no tratada conlleva un mal pronóstico con una edad promedio de supervivencia de 35 años; con $75 \%$ de mortalidad a los 46 años. "Las complicaciones a largo plazo son la consecuencia de la hipertensión, incluida la enfermedad arterial coronaria prematura, accidente cerebrovascular, endocarditis, disección aórtica e insuficiencia cardíaca" (Salazar, 2013). Además, la coartación recurrente y la formación futura de un aneurisma pueden ocurrir después de una reparación quirúrgica y endovascular exitosa que exige una vigilancia estrecha a largo plazo.

\section{Metodología}

Esta investigación está enfocada en el estudio médico de la Coartación aortica, diagnostico y tratamiento. Por tanto, se trata de una revisión bibliográfica vigente y de toda documentación importante al respecto.

La revisión se ha centrado en textos, documentos y artículos científicos publicados y disponibles en la web, considerando que aquella herencia de la globalización permite acceder a mayor y mejor información a través de los instrumentos de tecnología. El motor de búsqueda ha sido herramientas académicas de la web que direccionan específicamente a archivos con validez y reconocimiento científico, descartando toda información no confirmada o sin las respectivas referencias bibliográficas.

\section{Resultados}

\section{Epidemiología}

La coartación de la aorta (CoA) "es el quinto defecto cardíaco congénito más común, y representa del 6 al $8 \%$ de los nacidos vivos con cardiopatía congénita, con una incidencia estimada de 1 de cada 2500 nacimientos" (Baumgartner, 2015). 
Afecta a más bebés varones que mujeres, con una proporción informada en varones de entre 1,27: 1 y 1,74: 1. Los pacientes con CoA pueden tener otros defectos como defecto del tabique auricular (ASD), defecto del tabique ventricular (VSD), defecto del canal auriculoventricular (AVCD), válvula aórtica bicúspide (BAV), transposición de grandes arterias (TGA), conducto arterioso persistente (CAP) , síndrome del corazón izquierdo hipoplásico (Niaz, 2017).

La CoA a menudo coexiste con otras lesiones obstructivas del corazón izquierdo como la estenosis mitral, la estenosis subaórtica y la estenosis aórtica. Alrededor del 50-60\% de los pacientes con coartación de la aorta o arco aórtico interrumpido tienen VAB. En comparación con las lesiones del lado derecho. "Las obstrucciones cardíacas del lado izquierdo se observan con más frecuencia en hombres que en mujeres. Una afección genética que se asocia con un mayor riesgo de coartación de la aorta (12-35\%) es el síndrome de Turner" (Mayo Clinic, 2017). Sin embargo, la etiología de la CoA no se comprende bien y se cree que se ve afectada por varios factores, incluido un componente genético, factores ambientales y arteriopatía.

\section{Anatomía de la coartación aortica}

Aunque la mayoría de los pacientes tienen un estrechamiento discreto de la aorta descendente en la inserción del conducto arterioso, "existe un espectro de estrechamiento aórtico que abarca las habituales lesiones torácicas discretas, defectos de segmento largo, hipoplasia tubular y, raramente, coartación localizada en la aorta abdominal" (Hope, 2010). En términos simples, la coartación se caracteriza por un estrechamiento discreto de la aorta torácica adyacente al ligamento arterioso.

Es importante destacar que "la coartación discreta es una aortopatía que se encuentra dentro de un espectro de anomalías del arco que van desde un estrechamiento discreto hasta un segmento largo de hipoplasia del arco" (Brown, 2013). Morfológicamente aparece como una plataforma localizada en la pared aórtica posterolateral. De acuerdo con Mann, (2015), existen "algunas variaciones anatómicas de la coartación. Puede aparecer como (a) estrechamiento discreto (b) hipoplasia tubular de cualquier parte del arco o (c) interrupción del arco aórtico".

La CoA también se ha descrito como una arteriopatía difusa con anomalías en las propiedades elásticas de la aorta. Se ha informado de un aumento del colágeno y una disminución del componente del músculo liso de la aorta previa a la coartación. Aunque la CoA puede ser una enfermedad coronaria aislada, "también se encuentra comúnmente en otros síndromes congénitos y anomalías cardiovasculares. Por lo tanto, se debe realizar una investigación deliberada de la presencia de coartación en estos pacientes. La malformación cardiovascular más común asociada con CoA es BAV" (Johnson, 2001).

\section{Fisiopatología y presentación}

La presentación clínica de la coartación difiere significativamente en los pacientes pediátricos en comparación con los adultos. Aunque los lactantes con coartación grave pueden presentar signos y síntomas de insuficiencia cardíaca y shock cardiogénico a medida que se cierra el conducto, la mayoría de los adultos con coartación no reparada generalmente son asintomáticos.

Una presentación común de la coartación es la hipertensión arterial sistémica. "En adultos jóvenes que presentan hipertensión grave de las extremidades superiores, debe excluirse la coartación. Los pacientes que presentan hipertensión grave pueden experimentar síntomas que incluyen angina, dolor de cabeza, epistaxis e insuficiencia cardíaca" (Brown, 2013). "La coartación causa hipertensión de las extremidades su-

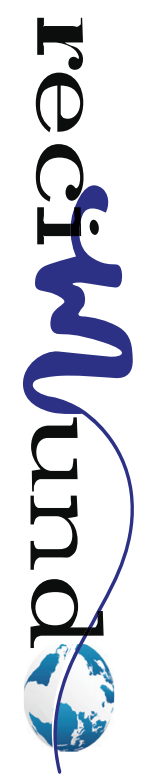


periores, que conduce a hipertensión sistémica e hipertrofia ventricular izquierda. Se han propuesto varios mecanismos para la hipertensión en pacientes con coartación, que incluyen distensibilidad arterial reducida, sensibilidad barorreceptora atenuada y disfunción endotelial" (Kaya, 2018)

La edad en el momento de la reparación es un determinante importante del desarrollo de hipertensión tardía. Los pacientes que reciben la reparación en la infancia tienen menos del $5 \%$ de probabilidades de desarrollar hipertensión en la edad adulta temprana, mientras que los operados después de un año tienen una probabilidad del 25$33 \%$ de desarrollar hipertensión. La hipertensión tardía se asocia con obstrucción residual. No obstante, algunos pacientes con una reparación satisfactoria aún pueden desarrollar hipertensión tardía debido a una disfunción vascular.

En los recién nacidos a término, una de las causas importantes de insuficiencia cardíaca congestiva es la coartación aórtica. Más allá del período neonatal, la mayoría de los pacientes son asintomáticos y presentan hipertensión difícil de controlar en los años posteriores. En adultos no diagnosticados previamente, el signo de presentación clásico es la hipertensión. Los pacientes mayores pueden quejarse de dolores de cabeza, fatiga de las piernas con el ejercicio y extremidades frías.

La coartación puede ser parte de síndromes como Síndrome de Turner, síndrome de Williams o el complejo de Shone. "Casi el 50\% de los casos están asociados a BAV. Otras anomalías asociadas incluyen aneurismas intracraneales (más comúnmente del círculo de Willis) en el 2-10\% de los casos y aneurismas adquiridos de la arteria intercostal" (de Divitiis, 2001). Las causas de muerte incluyen insuficiencia cardíaca, rotura aórtica, disección aórtica, endocarditis, endarteritis, hemorragia intracerebral e infarto de miocardio. Los pacientes con
BAV asociado también pueden desarrollar estenosis aórtica significativa, insuficiencia aórtica y aorta ascendente dilatada por degeneración mixomatosa de la pared medial de la aorta.

\section{Examen físico}

En la exploración física, los pulsos arteriales femorales están disminuidos y suelen retrasarse. En raras ocasiones, se puede informar claudicación debido a isquemia de las extremidades inferiores.

La auscultación del borde esternal izquierdo puede demostrar un soplo sistólico intenso con radiación en la espalda. Puede palparse un frémito asociado en la escotadura supraesternal. Si se ha desarrollado una sobrecarga de volumen o presión del ventrículo izquierdo, puede haber una elevación del ventrículo izquierdo. El hallazgo de un soplo continuo puede sugerir la presencia de colaterales arteriales en aquellos con coartación significativa no reparada de larga duración (Mann, 2015).

Si se sospecha coartación aórtica, se debe medir la presión arterial en ambos brazos y piernas en posición supina. Normalmente, la PA en las extremidades inferiores es un 10-20\% más alta que la extremidades superiores debido a la amplificación de ondas. "Si la PA en la pierna es más baja que la PA del brazo en 10 mmHg o más, se debe sospechar una coartación. Un gradiente de presión de $35 \mathrm{mmHg}$ o más se considera muy específico para la coartación" (Marx, 2006). La presencia de vasos colaterales puede disminuir el gradiente de presión. También se pueden palpar las pulsaciones arteriales de las colaterales a las arterias intercostales e interescapulares.

\section{Evaluación diagnóstica}

\section{Evaluación inicial}

El electrocardiograma de un paciente con 
coartación puede ser normal o mostrar evidencia de hipertrofia ventricular izquierda por sobrecarga crónica de presión ventricular izquierda. "En la radiografía de tórax, un signo en "figura de tres" formado por el nudo aórtico, el segmento estenótico y el segmento postestenótico dilatado de la aorta sugiere CoA" (Hope, 2010). El borde del corazón puede ser normal o estar levemente agrandado. "También se puede observar una muesca costal inferior en la tercera a la octava costillas causada bilateralmente por la presencia de arterias colaterales intercostales dilatadas" (de Divitiis, 2001).

\section{Ecocardiografía}

La ecocardiografía transtorácica es la más accesible y la principal estancia para el médico en ejercicio. Se recomienda un ecocardiograma completo en la evaluación inicial de un paciente con CoA reparada o sospechada. Además de la caracterización de la coartación en sí, es importante evaluar "la evidencia de sobrecarga de volumen o presión ventricular izquierda, hipertrofia ventricular izquierda, tamaño y disfunción sistólica y diastólica del ventrículo izquierdo" (Brown, 2013). Debe prestarse especial atención a la identificación de los defectos cardíacos asociados, especialmente las lesiones del lado izquierdo, así como también, la morfología de la válvula aórtica y la evidencia de estenosis aórtica subvalvular, valvular y supravalvular.

Las dimensiones de la raíz aórtica y la aorta ascendente pueden seguirse en serie para evaluar la presencia de aortopatía asociada.

Las ventanas supraesternales son importantes para ver el arco aórtico desde el eje largo, en imágenes bidimensionales y mediante Doppler de flujo en color. La visualización del arco aórtico en el eje largo puede demostrar un área focal de estrechamiento de la aorta torácica distal al despegue de la arteria subclavia izquierda con turbulencia de flujo asociada en el Doppler de flujo en color (Hope, 2010).

Se obtienen imágenes de la coartación desde las proyecciones paraesternal izquierda superior con angulación lateral de la sonda hacia el hombro izquierdo. La proyección de la muesca supraesternal se utiliza para obtener el gradiente Doppler (Figuras 1 y 2). Las imágenes subcostales se utilizan para evaluar la región torácica distal y superioraorta abdominal. El segmento estenótico puede ser discreto, segmentario o largo, por lo que se debe obtener una imagen de todo el arco aórtico, en particular el origen de la arteria subclavia izquierda, ya que en la hipoplasia del arco transversal la distancia entre el origen de la arteria carótida común izquierda y la arteria subclavia izquierda puede ser aumentado. Las imágenes de baja frecuencia y las imágenes armónicas pueden mejorar la calidad de la imagen (Houston, 2007).

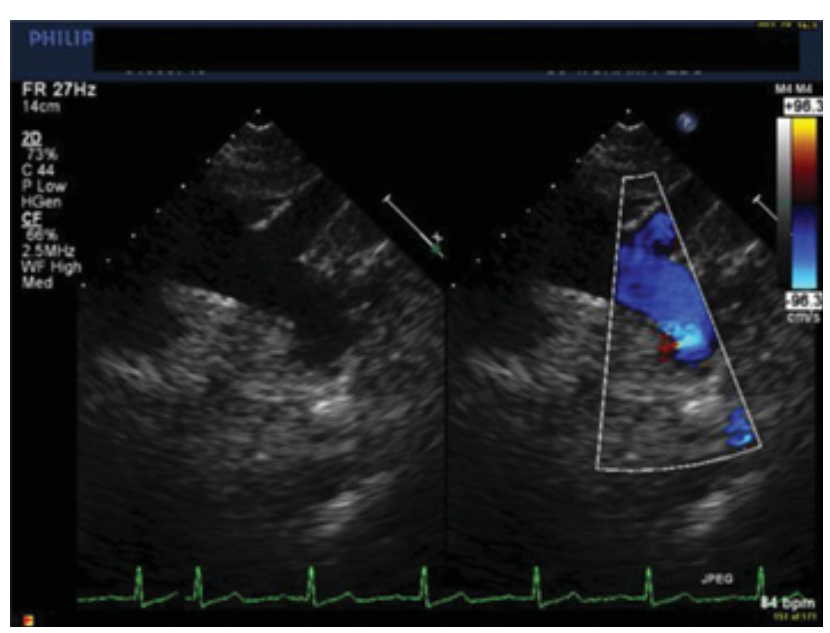

Figura 1. Imagen de eco transtorácica 2D que muestra coartación de la aorta distal a la arteria subclavia izquierda.

Fuente: (Houston, 2007). 


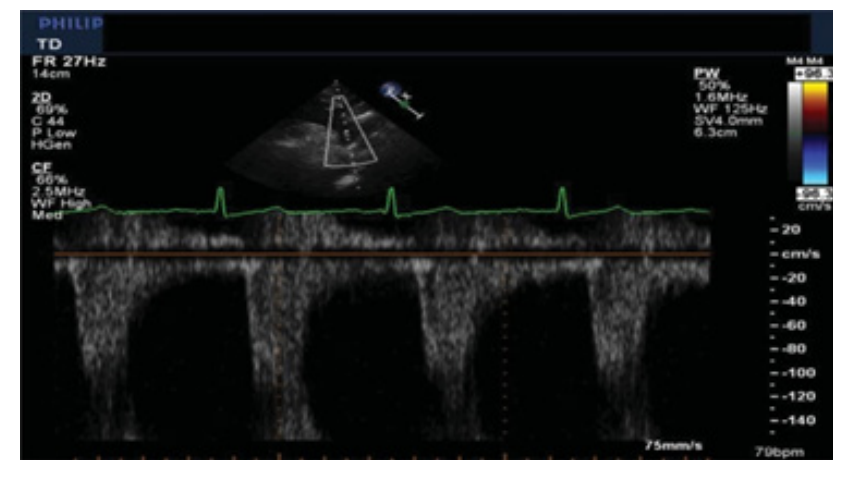

Figura 2. Perfil Doppler de onda de pulso a través del segmento de coartación que demuestra un gradiente de presión.

Fuente: (Houston, 2007).

En la formación de imágenes Doppler, el alias de flujo de color se vería en y más allá del segmento estrecho. "Aumenta la velocidad sistólica en la aorta descendente. Si hay hipoplasia del arco transversalla velocidad proximal también aumenta, por lo que el gradiente de presión sistólica debe calcularse con la ecuación 4 de Bernoulli ampliada (V22-V12)" (Houston, 2007). En casos graves, hay un gradiente durante la sístole y la diástole a través de la estenosis, lo que da como resultado el patrón clásico de diente de sierra.

La presencia de arterias colaterales puede hacer que el doppler subestime la gravedad de la obstrucción. Algunos de los otros factores que pueden afectar el gradiente Doppler incluyen obstrucción severa, vasos largos y tortuosos o gradiente excéntrico. Sin embargo, con la coartación de larga duración, es posible que se hayan desarrollado colaterales importantes, reduciendo así el gradiente sistólico máximo a través del sitio de la estenosis. "Un patrón en forma de diente de sierra que se observa en el Doppler de onda continua refleja el flujo directo persistente en la diástole debido a la escorrentía diastólica" (Hope, 2010).

En ausencia de obstrucción proximal cuando el doppler de la onda de pulso se coloca en la aorta abdominal, la forma de onda muestra un golpe sistólico ascendente rápido, un tiempo de desaceleración corto, seguido de una breve inversión del flujo diastólico temprano y poco flujo anterógrado durante la diástole.

En presencia de coartación hay una pérdida de la inversión del flujo diastólico temprano, que es muy sensible.para la detección de obstrucciones aguas arriba. La velocidad sistólica se reduce, hay un flujo anterógrado continuo y un aumento de la velocidad del flujo diastólico. Si el retraso entre la onda $R$ en el ECG y la velocidad máxima de la aorta abdominal es $>50 \mathrm{~ms}$, se asocia con coartación (Nielsen, 2005).

\section{Imagen de resonancia magnética}

La resonancia magnética (MRI) es el método más completo para evaluar la coartación de la aorta. La resonancia magnética no expone a los pacientes a radiaciones ionizantes, lo cual es una consideración importante para los pacientes jóvenes que tendrían que someterse a imágenes en serie. La resonancia magnética cardíaca (cMRI) se ha convertido en una valiosa modalidad no invasiva para evaluar a los pacientes con coartación reparada y no reparada. En adultos con una ventana de imagen ecocardiográfica subóptima, se puede utilizar cMRI para caracterizar la válvula aórtica, la raíz aórtica, el tamaño y la función del ventrículo izquierdo.

La cMRI, junto con la angiografía por resonancia magnética mejorada con gadolinio, proporciona una excelente resolución de la anatomía cardíaca y las estructuras vasculares. En comparación con la ecocardiografía, la RMNc demuestra una visualización superior del arco aórtico con una caracterización precisa de la ubicación y extensión de la coartación. y evaluación de la presencia y extensión de vasos colaterales (Eckhauser, 2015). 
En el paciente no reparado, el área transversal aórtica mínima medida y el tiempo de desaceleración corregido por la frecuencia cardíaca en la aorta descendente pueden usarse para predecir un gradiente significativo mediante cateterismo cardíaco y la necesidad futura de intervenciones. "La cMRI proporciona una visualización excepcional del arco aórtico y la detección de complicaciones posteriores a la reparación, incluidos los "retorcimientos" del arco y los pseudoaneurismas" (Kaya, 2018)

La angiografía por resonancia magnética de la aorta torácica también permite evaluar la dilatación postestenótica o la formación de aneurismas en el sitio de una reparación previa. "Es importante destacar que la falta de radiación ionizante proporciona una ventaja de la RMc sobre la TC, en la evaluación seriada de las complicaciones tardías después de la reparación" (Eckhauser, 2015).

Con las imágenes de contraste de fase se puede determinar el grado de flujo colateral. El volumen de flujose evalúa en la aorta próxima a la estenosis y luego a nivel del diafragma. Por lo general, "hay una disminución del $7 \%$ en el flujo total desde la aorta proximal a la distal; si hay un aumento del flujo del 5\% o más, es muy indicativo de que el flujo colateral se une a la aorta descendente" (Hope, 2010). La RM de flujo en cuatro dimensiones es una herramienta emergente para evaluar la importancia hemodinámica del flujo sanguíneo colateral (Figura 3).

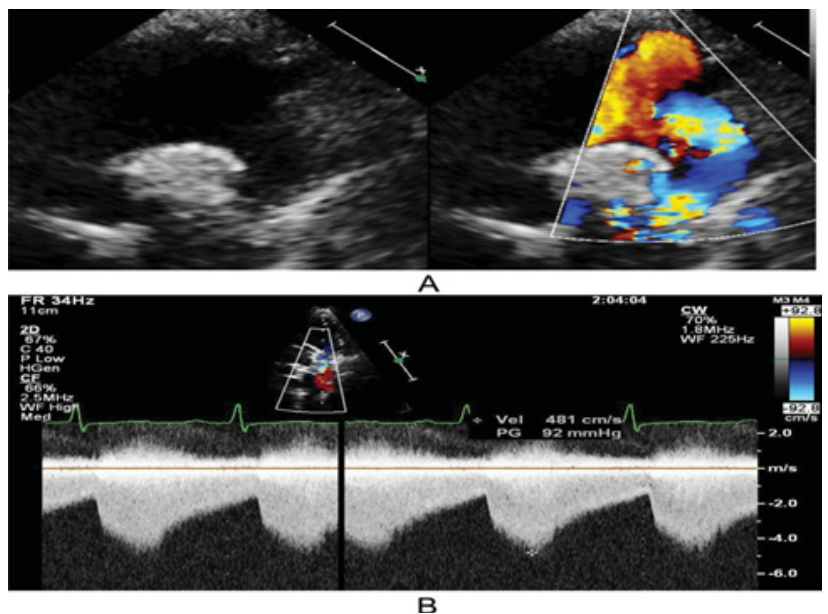

Figura 3. (A) Eco 2D con Doppler de flujo color que muestra un estrechamiento severo de la aorta descendente proximal con turbulencia significativa y una velocidad máxima de 4,8 m / s compatible con coartación aórtica grave. (B) El trazado Doppler muestra retraso en el retorno a la línea de base en la diástole (arrastre diastólico) y embotamiento del patrón Doppler aórtico abdominal consistente con coartación aórtica significativa.

Fuente: (Hope, 2010)

\section{Tomografía computarizada angiografía (ATC)}

Aunque la cMRI es el modo preferido de seguimiento en serie para los pacientes después de la reparación de la coartación, se puede considerar el uso de TC cardiovascular en pacientes seleccionados.

En particular, la cMRI en pacientes con stents transcatéter puede tener un artefacto de susceptibilidad que impida una evaluación precisa de las complicaciones tardías asociadas con estas intervenciones. Con la RMc, los artefactos metálicos pueden dificultar la evaluación de la permeabilidad de la luz del vaso, identificando reestenosis, aneurisma o fractura del stent (Houston, 2007).

El uso de la TC elimina las preocupaciones sobre los artefactos metálicos que afectan

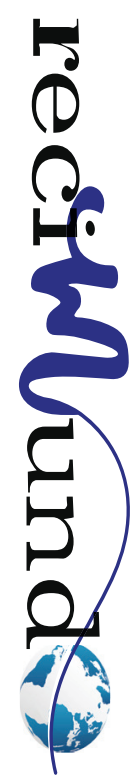


la evaluación precisa de los segmentos de la aorta con stent. Otras ventajas de la TC cardíaca sobre la RMc incluyen una resolución de imagen mejorada, un tiempo de exploración más corto y una mayor disponibilidad (figura $\mathrm{N}^{\circ} 4$ ).

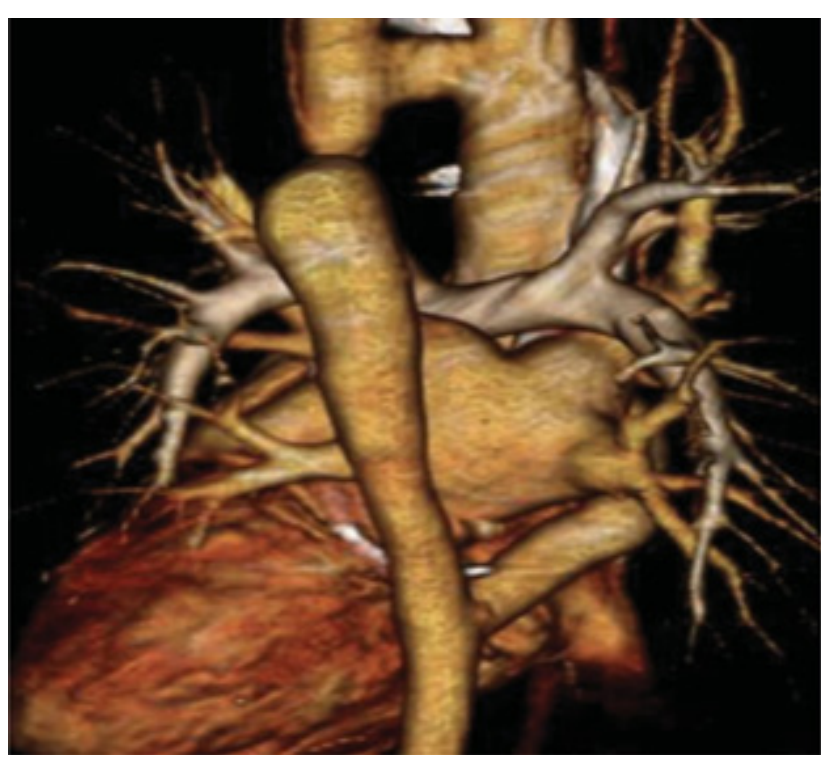

Figura 4. Reconstrucción 3D (angiografía por TC) que muestra un segmento discreto de CoA y una dilatación leve de la aorta torácica descendente distal al segmento de coartación

Fuente: (Houston, 2007)

La ATC también se utiliza para evaluar anomalías coronarias concomitantes que pueden no visualizarse bien con la RMc. "Los pacientes con marcapasos o desfibriladores automáticos implantables que no son compatibles con cMRI pueden beneficiarse de la vigilancia con ATC cardiovascular" (Hope, 2010). De manera similar a la RMC, se puede realizar una TC cardiovascular para obtener imágenes del segmento de coartación, "cualquier dilatación aneurismática distal al segmento de coartación, reparación posterior a la recoartación, hipoplasia del arco aórtico, siguen dimensiones aórticas seriadas y también pueden mostrar anomalías vasculares asociadas, como vena cava superior doble o grandes vasos aberrantes" (Hope, 2010).
La formación de vasos colaterales también se puede visualizar con ATC. La principal desventaja de la ATC es la exposición a la radiación, por lo que los algoritmos de ahorro de dosis son muy importantes para reducir la exposición de los pacientes a la radiación.

\section{Administración y tratamiento}

En 2008, las directrices del American College of Cardiology y la American Heart Association (ACC / AHA) para adultos con cardiopatías congénitas recomendaron la intervención de la coartación en las siguientes indicaciones:

a. Gradiente de coartación pico a pico $\geq 20 \mathrm{mmHg}$. El gradiente de pico a pico es una medida derivada de los datos de cateterismo en la que la presión máxima más allá de la coartación se resta de la presión máxima próxima a la coartación.

b. Gradiente de coartación de pico a pico <20 mmHg con evidencia de imagen anatómica de coartación significativa y evidencia radiológica de flujo colateral significativo.

Hipertensión sistémica, enfermedad coronaria acelerada, accidente cerebrovascular, disección aórtica y corazón, son complicaciones frecuentes en adultos que no se han sometido a la corrección de su coartación o que fueron operados más tarde en la vida. La reparación de la coartación después de la primera infancia no previene la persistencia o la recurrencia tardía de la hipertensión sistémica.

Como resultado, la corrección de la coartación debe realizarse en la infancia o la niñez temprana para prevenir el desarrollo de hipertensión sistémica crónica. Si la coartación escapa a la detección temprana, la reparación debe realizarse en el momento del diagnóstico posterior si está clínicamente indicado. El manejo con medicamentos antihipertensivos es importante para preve- 
nir complicaciones a largo plazo (Kenny \& Hijazi, 2011).

Según las directrices, los medicamentos de primera línea en el tratamiento de la hipertensión en pacientes con CoA son los inhibidores de la enzima convertidora de angiotensina (ECA), los bloqueadores del receptor de angiotensina (BRA) y los bloqueadores beta (BB). "La hipertensión puede tratarse con tratamiento médico, pero la coartación o recoartación de la aorta debe repararse de forma percutánea o quirúrgica" (Kenny \& Hijazi, 2011).

La elección de una intervención sobre otra depende del paciente individual y debe hacerse en colaboración con un equipo interdisciplinario que incluya un cardiólogo, intervencionista y cirujano de cardiopatías congénitas en adultos (ACHD) con formación en ACHD. "Por ejemplo, es más probable que los pacientes con un segmento largo de coartación de la aorta, una anatomía compleja del arco o con interrupción de la aorta necesiten una cirugía a corazón abierto en lugar de una intervención transcatéter" (Feltes, 2011).

\section{Intervención percutánea}

A mediados de la década de 1900, la reparación de la coartación de la aorta era completamente quirúrgica. La angioplastia con balón es una alternativa percutánea a la reparación quirúrgica para bebés mayores y niños pequeños (mayores de 4 meses) con coartación discreta nativa. Sigue siendo la intervención preferida para todos los pacientes con recoartación aislada independientemente de la edad.

Sin embargo, la colocación de un stent ha reemplazado a la angioplastia con balón como el procedimiento de elección en niños mayores y adultos con coartación nativa. Actualmente, la dilatación con balón y la colocación de stents siguen siendo las intervenciones transcatéter que pueden utili- zarse para el tratamiento de la CoA (Fawzy, 2018).

Aunque la angioplastia con balón fue el tratamiento de elección para la coartación nativa discreta en adultos en el pasado, la mayoría de los centros realizan actualmente la implantación de stents para niños mayores y adultos con coartación nativa discreta o de segmento largo. A lo largo de los años, los continuos avances en la tecnología y las técnicas basadas en catéteres han hecho disponible una variedad de posibilidades de intervención percutánea. Las mejoras en el campo han permitido que las intervenciones evolucionen desde la angioplastia con balón hasta los stents endovasculares y los stents cubiertos.

\section{Quirúrgico}

Las técnicas de uso común para el tratamiento de la CoA en el período infantil son la resección y la anastomosis término-terminal directa o la arterioplastia con colgajo subclavio, esto se debe a que las condiciones anatómicas son más favorables. La arterioplastia con colgajo subclavio y la aortoplastia con injerto de parche "se han desarrollado como una alternativa a la resección y la anastomosis término-terminal directa en las que más de la mitad de los pacientes experimentan problemas de reacoartación de inicio tardío" (Ozbaydar, 2007). Sin embargo, la CoA en adolescentes y adultos a menudo se complica con la aparición de comorbilidades asociadas como aneurismas aórticos, disecciones, valvulopatía aórtica y otras enfermedades cardiovasculares.

Los estudios demuestran que tener un BAV es un factor de riesgo de mortalidad. Lo más probable es que esto se deba a que "Ios BAV se han asociado con insuficiencia aórtica y estenosis además de la dilatación y disección de la aorta, lo que ha dado lugar a una posible necesidad de cirugía a corazón abierto" (Ozbaydar, 2007) 
El principal objetivo del tratamiento quirúrgico en la CoA es la eliminación de la estenosis. "La técnica quirúrgica se selecciona de acuerdo con la longitud del segmento coartado, la localización con el ductus, el estado de la circulación colateral en la aorta distal y las alteraciones ateroscleróticas en la pared aórtica" (Ozbaydar, 2007).

Cuando la reparación quirúrgica de la coartación se realiza a una edad posterior, debe tenerse en cuenta la posibilidad de estas comorbilidades cardiovasculares. "Algunos de los otros riesgos en la cirugía a considerar están relacionados con lesiones isquémicas espinales y hemorragia intraoperatoria por grandes cantidades de colaterales" (Baumgartner, 2015). En general, se ha demostrado que la reparación quirúrgica de la CoA tiene una tasa de mortalidad baja. "Sin embargo, a medida que estos pacientes continúan en seguimiento con sus cardiólogos, se observa una nueva coartación a largo plazo, adicionalmente, aparte de los procedimientos percutáneos adicionales, estos pacientes a veces necesitan ser evaluados para intervenciones quirúrgicas adicionales" (Brown, 2013).

\section{Conclusión}

Durante el desarrollo del proceso investigativo se evidencia la importancia y lo delicada que es para los pacientes que presentan este tipo de afección tener un seguimiento inmediato una vez diagnosticada la coartación aortica aunado a los pacientes con CoA que se han sometido a reparación requieren vigilancia de por vida.

Dado que este tipo de cardiopatía coronaria se asocia con muchas complicaciones a largo plazo, se recomienda el tratamiento colaborativo por parte de cardiólogos con experiencia en la cardiopatía coronaria en adultos. Esto se debe a que hoy en día, los pacientes con cardiopatía coronaria sobreviven hasta la edad adulta, en un promedio del 5 al $8 \%$, por lo cual resulta de mucha importancia comprender la anatomía, la fisiopatología y el tratamiento necesario para el abordaje de estas personas.

Aunque la ecocardiografía es una herramienta fundamental en la evaluación de los pacientes después de la reparación de la coartación, generalmente, se necesitan imágenes avanzadas para una evaluación completa. La cMRI es la modalidad de imagen preferida para CoA reparada y no reparada. Alternativamente, la TC cardiovascular es la más adecuada para evaluar a los pacientes con stents endovasculares o aquellos con contraindicaciones para la RMc. No es raro que se desarrollen complicaciones o se requieran intervenciones percutáneas o quirúrgicas adicionales durante la vida del paciente. Por tal motivo, se enfatiza la importancia de la atención de seguimiento a largo plazo, especialmente en un centro especializado en la atención de pacientes con cardiopatías congénitas.

\section{Bibliografía}

Baumgartner, H. (2015). ESC guidelines for the management of grown-up congenital heart disease. European Heart Journal, 2915-2957.

Brown, M. (2013). Coarctation of the aorta: Lifelong surveillance is mandatory following surgical repair. Journal of the American College of Cardiology , 1020-1025.

de Divitiis, M. (2001). Vascular dysfunction after repair of coarctation of the aorta: Impact of early surgery. Circulation , |165-|1170.

Eckhauser, A. (2015). Turner syndrome in girls presenting with coarctation of the aorta. The Journal of Pediatrics , 1062-1066.

Fawzy, M. (2018). Twenty-two years of follow-up results of balloon angioplasty for dis- creet native coarctation of the aorta in adolescents and adults. American Heart Journal, 910-917.

Feltes, T. (2011). Indications for cardiac catheterization and intervention in pediatric cardiac disease: A scientific statement from the American Heart Association. Circulation, 2607-2652.

Hope, M. (2010). Clinical evaluation of aortic coarctation with 4D flow MR imaging. Journal of Magnetic Resonance Imaging , 711-718. 
Houston, A. (2007). Doppler ultrasound in the assessment of severity of coarctation of the aorta and interruption of the aortic arch. British Heart Journal , 38-43.

Johnson, D. (2001). Resetting of the cardiopulmonary baroreflex 10 years after surgical repair of coarctation of the aorta. Heart, 318-325.

Kaya, U. (2018). Surgical management of aortic coarctation from infant to adult. The Eurasian journal of medicine, 14-18.

Kenny, D., \& Hijazi, Z. (2011). Coarctation of the aorta: From fetal life to adulthood. Cardiology Journal , 487-495.

Mann, D. (2015). Braunwald's Heart Disease : A Textbook of Cardiovascular Medicine. Elsevier, Saunders.

Marx, G. A. (2006). Accuracy and pitfalls of Doppler evaluation of the pressure gradient in aortic coarctation. Journal of the American College of Cardiology , 1379-1385.
Mayo Clinic. (2017, Nov 18). Sindrome de Turner. Retrieved Mar 30, 2021, from https://www.mayoclinic. org/es-es/diseases-conditions/turner-syndrome/ symptoms-causes/syc-20360782

Niaz, T. (2017). Incidence, morphology, and progression of bicuspid aortic valve in pediatric and young adult subjects with coexisting congenital heart defects. Congenital Heart Disease, 261-269.

Nielsen, J. (2005). Magnetic resonance imaging predictors of coarctation severity. Circulation , 622628.

Ozbaydar, M. (2007). Arthroscopic reconstruction of the rotator cuff. The current gold standard? Orthopade , 825-833.

Salazar, I. (2013). Cardiopatias Congenitas. ENEO , 71.

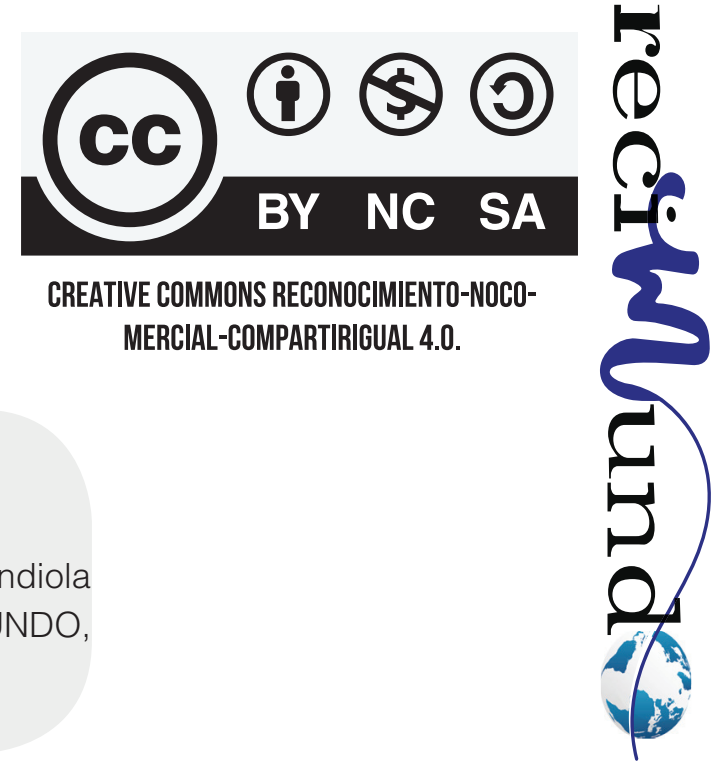

\section{CITAR ESTE ARTICULO:}

Reinoso Trujillo, K. A., Fabregas Almanza, R., Mendoza Niño, F. A., \& Mindiola Joly, D. (2021). Coartación aortica, diagnóstico y tratamiento. RECIMUNDO, 5(2), 4-15. https://doi.org/10.26820/recimundo/5.(2).abril.2021.4-15 